\title{
Transmission Performance Improvement Using Broadband Incoherent Counter-Pumped Distributed Raman Amplification
}

\author{
Md Asif Iqbal, Mingming Tan, and Paul Harper \\ Aston Institute of Photonic Technologies, Aston University, B4 7ET, Birmingham, UK \\ iqbalm7@aston.ac.uk
}

\begin{abstract}
We propose a novel dual-order counter-pumped distributed Raman amplification technique using broadband incoherent $1^{\text {st }}$-order pump to suppress RIN transfer and improve Qfactor and transmission reach by $0.3 \mathrm{~dB}$ and $833 \mathrm{~km}$ respectively compared with conventional narrowband pumping.

OCIS codes: (060.4510) Optical communications; (060.2320) Fibre optics amplifiers and oscillators;
\end{abstract}

\section{Introduction}

In this paper, we experimentally demonstrate a novel dual-order counter pumped distributed Raman amplification (DRA) technique using a broadband $1^{\text {st }}$-order pump to mitigate relative intensity noise (RIN) transfer even in counter-pumping and improve transmission performance compared with conventional Raman amplifiers.

DRA shows better optical signal to noise ratio (OSNR) than conventional lumped amplification i.e. erbium doped fibre amplifier (EDFA) [1]. Although bidirectional DRA provides improved OSNR counter-pumping is preferred in long-haul transmission system to avoid the fundamental problem of RIN transfer from co-pump(s) to signal [2]. The signal RIN penalty is very low in counter-pumping DRA due to the averaging over longer span length and lower cut-off frequency of the RIN transfer function [3]. However, this small amount of RIN transfer over 10-100s of kHz from high power and high RIN pump(s), can still limit the transmission performance.

In this paper, we present a dual-order counter-pumping scheme which uses an incoherent broadband $1^{\text {st }}$-order pump. This pump improves the ASE noise performance by distributing the gain further into the amplifier span and suppress the signal RIN penalty by mitigating the evolution of RIN transfer from higher order $2^{\text {nd }}$-order pump to signal. Here, the dual-order counter-pumping consists of a $1365 \mathrm{~nm} 2^{\text {nd }}$-order fibre laser and a broadband $1455 \mathrm{~nm}$ $1^{\text {st }}$-order seed, generated using Rayleigh scattering. We show that, the use of inherently depolarized $1^{\text {st }}$-order broadband pump allows improved transmission performance by reducing the signal RIN penalty compared with conventional $11^{\text {st }}$-order Raman pumping schemes which use low RIN semiconductor pumps or random fibre laser (RFL) pump with narrow bandwidth profiles. In a $10 \times 120 \mathrm{~Gb} / \mathrm{s}$ DP-QPSK wavelength division multiplexed (WDM) system, our proposed pumping scheme extends the transmission reach up to $7915 \mathrm{~km}$ with $833 \mathrm{~km}$ transmission reach improvements compared with conventional Raman pumping schemes. In addition, the use of such broadband pumping can provide a flatter gain spectrum, which reduces the number of pumps required in broadband (i.e. $\mathrm{C}$ and L band) transmission.

\section{Experimental setup and distributed Raman span characterization}

We investigated three dual-order counter-pumped DRA schemes with $\sim 83 \mathrm{~km}$ standard single mode fibre (SSMF) which include a commercially available depolarized $1365 \mathrm{~nm}$ Raman pump laser with $-113 \mathrm{~dB} / \mathrm{Hz} \mathrm{RIN}$ as $2^{\text {nd }}$-order counter-pump and different $1^{\text {st }}$-order sources: random fibre laser (RFL) based pump (scheme-1), proposed broadband pump (scheme-2) and commercial semiconductor pump (scheme-3) as shown in Fig. 1(b-d) respectively. In scheme-2, the broadband $1^{\text {st }}$-order pump seed at $1455 \mathrm{~nm}$ was generated by counter-pumping a separate $10 \mathrm{~km}$ SSMF by another $2^{\text {nd }}$-order $1365 \mathrm{~nm}$ pump at $3.5 \mathrm{~W}$ power in an open cavity configuration [4]. This $1^{\text {st }}$ order seed was then amplified with the residual $1365 \mathrm{~nm}$ pump to launch $20 \mathrm{~mW}$ of $1455 \mathrm{~nm}$ broadband pump into the main amplifier span. The length of SSMF in the broadband seed generation section was optimized for efficient generation of stable $1455 \mathrm{~nm}$ broadband pump power. The $3 \mathrm{~dB}$ bandwidth and RIN level of the pump were measured as $\sim 12 \mathrm{~nm}$ and $-132 \mathrm{~dB} / \mathrm{Hz}$ respectively. In scheme-3, two $1455 \mathrm{~nm}$ semiconductor laser diodes with $0.8 \mathrm{~nm} 3 \mathrm{~dB}$ bandwidth and $135 \mathrm{~dB} / \mathrm{Hz}$ RIN were depolarized through a polarization beam combiner (PBC) and used as $1^{\text {st }}$-order pump as depicted in Fig. 1(d). The $1^{\text {st }}$-order pump in scheme- 2 and 3 was combined with the $2^{\text {nd }}$-order $1365 \mathrm{~nm}$ pump and then to the amplifier span through a $3 \times 1$ WDM coupler. An isolator was also used at the $1455 \mathrm{~nm}$ pump output to restrict the back-propagated pumps. Fixed $20 \mathrm{~mW}$ and $1 \mathrm{~W}$ powers of $1^{\text {st }}$ and $2^{\text {nd }}$ order counter-pumps were used respectively in both scheme- 2 and 3 to maintain $0 \mathrm{~dB}$ net loss across the $\sim 83 \mathrm{~km}$ Raman span. 


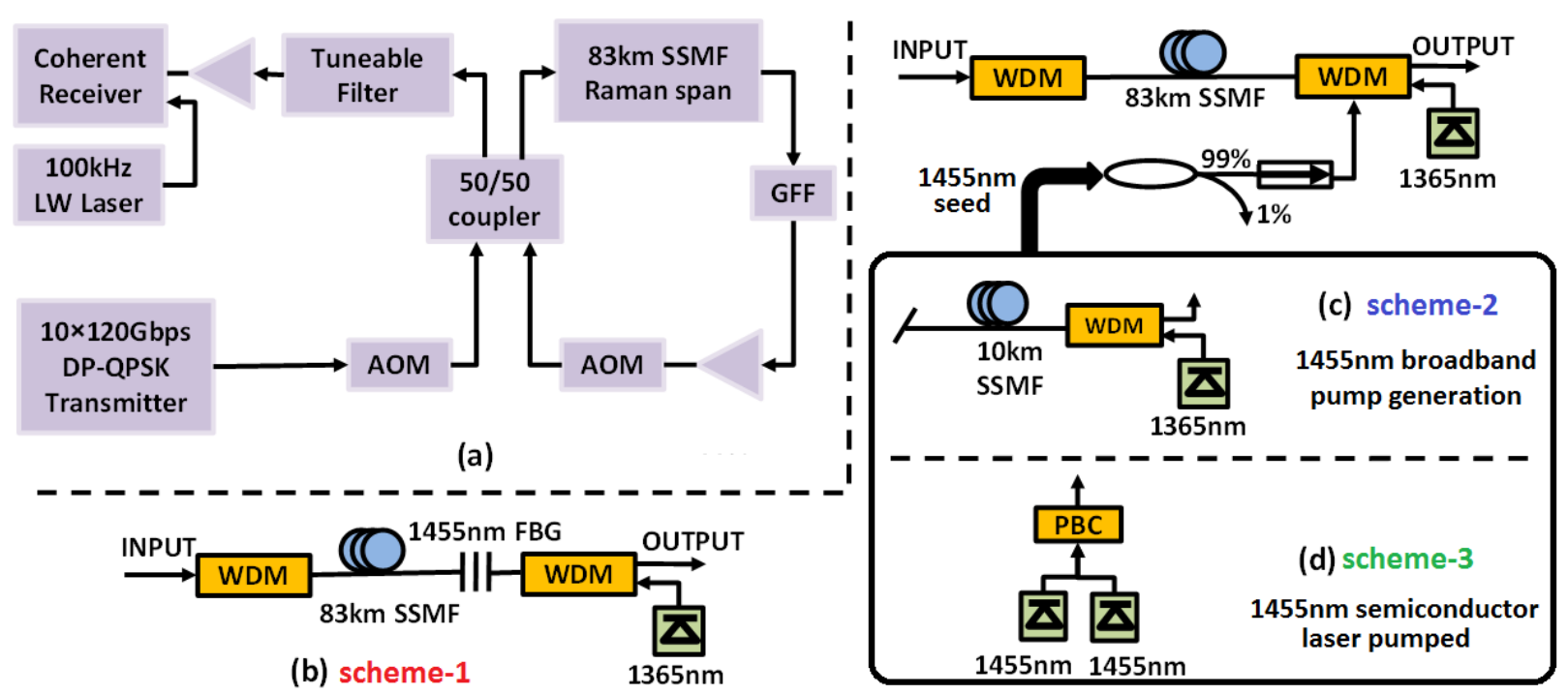

Fig. 1. (a) Coherent transmission experiment setup in a recirculating loop with $83 \mathrm{~km}$ DRA span; and different dual order counter-pumped only distributed Raman amplification schemes consisting of a $2^{\text {nd }}$ order $1365 \mathrm{~nm}$ pump and a $1^{\text {st }}$ order $1455 \mathrm{~nm}$ pump based on: (b) random fibre laser (RFL) using a FBG; (c) proposed broadband pump and (d) semiconductor laser didoes.

Fig. 1(b) shows a RFL based dual order pumping (scheme-1) including a $1365 \mathrm{~nm}$ pump and a fibre Bragg grating (FBG) centred at $1455 \mathrm{~nm}$ (95\% reflectivity and $0.5 \mathrm{~nm} 3 \mathrm{~dB}$ bandwidth). The RFL at $1455 \mathrm{~nm}$ was formed in a distributed cavity created by the Rayleigh scattering and feedback from FBG [5,6] and the measured 3dB bandwidth and RIN level were $0.6 \mathrm{~nm}$ and $-115 \mathrm{~dB} / \mathrm{Hz}$ respectively. A $1365 \mathrm{~nm}$ pump power of $1.1 \mathrm{~W}$ was required to overcome the lasing threshold and maintain 16.5dB Raman on-off gain.

Comparisons of the spectral properties and RIN levels of different $1^{\text {st }}$-order pumps are shown in Fig. 2(a) and (b) respectively. The proposed broadband pump bandwidth is much wider (>10 times) than the other two schemes as shown in Fig. 2(a). In Fig. 2(b), semiconductor pump shows the lowest pump RIN level (-135dB/Hz). Broadband pump also has similar RIN (-132dB.Hz), whereas RFL pump shows $>15 \mathrm{~dB}$ increased RIN compared with others.

In order to verify the impact of RIN transfer in all the schemes, we maintained similar signal power profiles for all to ensure equal ASE noise performance. The signal power profiles were measured at $194 \mathrm{THz}(1545.32 \mathrm{~nm})$ signal. The pump powers used here provides similar profiles with 5.8 6dB SPVs across all three counter-pumped DRA schemes as shown in Fig. 2(c).

In these dual-order counter pumped DRAs, the main RIN transfer to the signal comes from the high RIN $1365 \mathrm{~nm}$ fibre laser. So the highest RIN transfer is expected from scheme- 1 with both the high RIN $1^{\text {st }}$ and $2^{\text {nd }}$-order pumps. First order semiconductor pumps based scheme-3 has the lowest $1^{\text {st }}$ order pump RIN (Fig. 2(b)) but narrow bandwidth profile still allows some RIN transfer from $2^{\text {nd }}$-order $1365 \mathrm{~nm}$ pump to signal. In scheme-2, the broadband incoherent $1^{\text {st }}$-order pump also has similar RIN as semiconductor pump, but RIN from higher order pump gets distributed over the wide bandwidth of $1^{\text {st }}$-order pump and averaged out due to the four-wave mixing effects, which subsequently ensures the lowest overall RIN transfer to the signal.

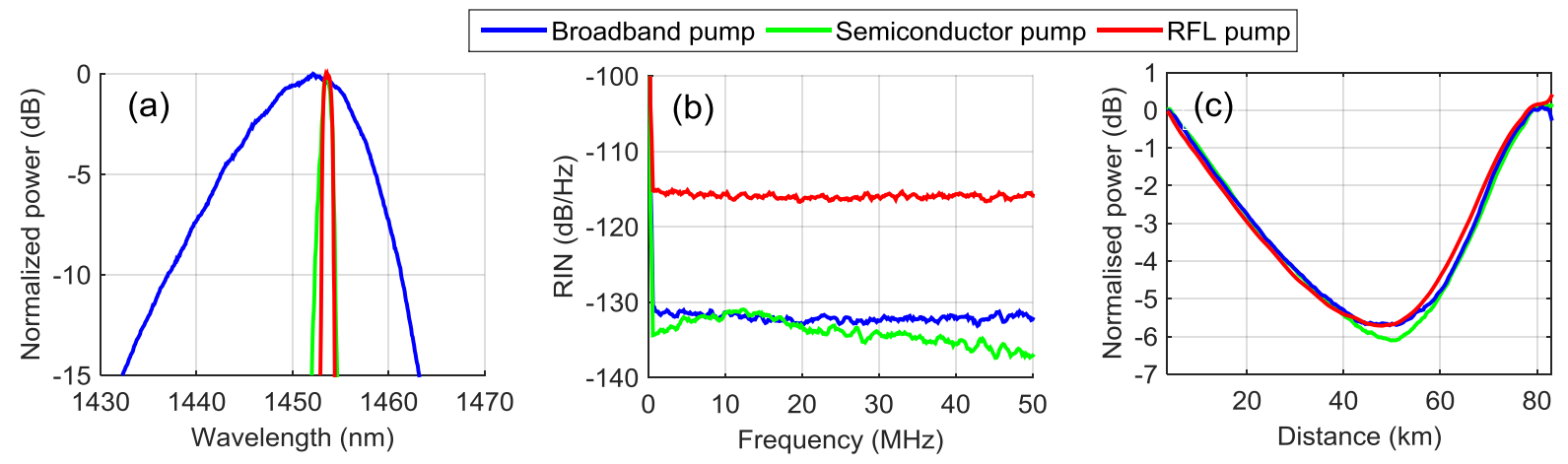

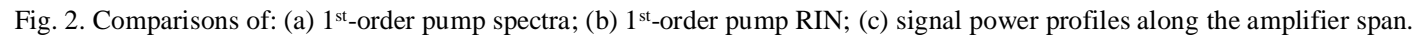




\section{Transmission results}

A long-haul coherent transmission setup in a recirculating loop with different dual-order counter-pumped DRA schemes is shown in Fig. 1(a). A typical $120 \mathrm{~Gb} / \mathrm{s}$ DP-QPSK transmitter was used with ten $100 \mathrm{GHz}$ spaced (193.4 194.3THz) WDM signals, combined with a tuneable 100kHz external cavity laser (ECL) as "channel under test". The total distributed Raman span loss was $\sim 17.6 \mathrm{~dB}$ including $16.5 \mathrm{~dB}$ from $\sim 83 \mathrm{~km}$ SSMF and $1.1 \mathrm{~dB}$ from the pair of pump/signal combiners at each end. The additional $\sim 12 \mathrm{~dB}$ passive loop loss from the gain flattening filter (GFF), acousto-optic modulator (AOM) and 50/50 coupler was compensated by a dual-stage EDFA at the end. In the standard polarization diverse coherent receiver, signal was first filtered out with a band pass tuneable filter and amplified by an EDFA before passing into an $80 \mathrm{GSa} / \mathrm{s}, 36 \mathrm{GHz}$ bandwidth oscilloscope. Offline digital signal processing (DSP) was used to post-process for the linear impairments and Q factors were calculated from the actual bit-error rate from 2 million bits. A Q-factor of $8.5 \mathrm{~dB}$ is also considered as a HD-FEC limit for maximum distance.

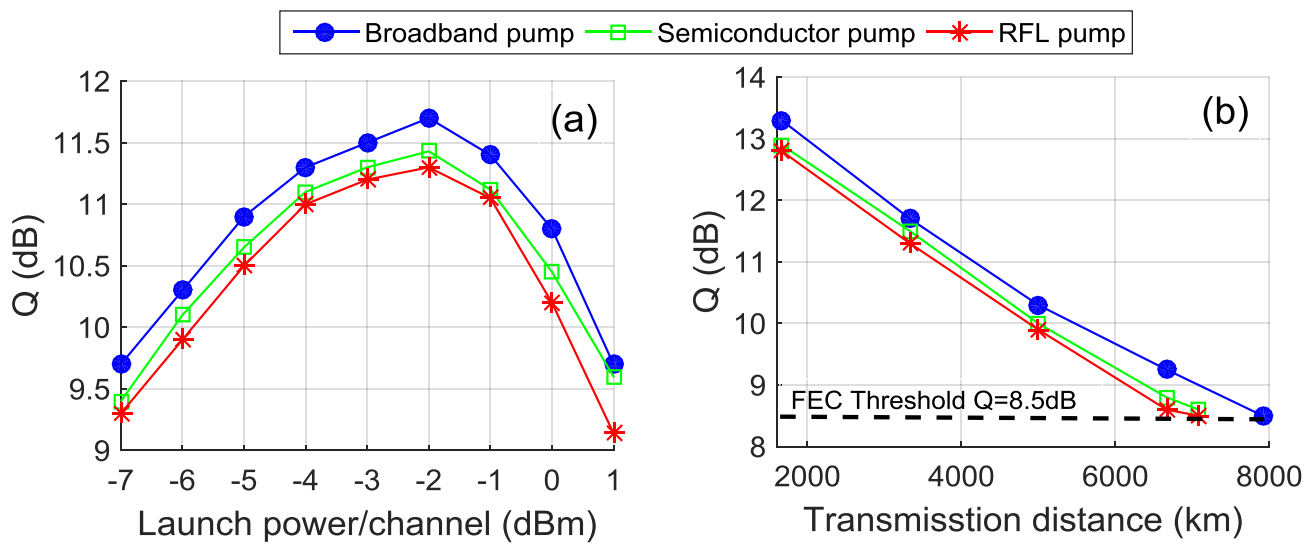

Fig. 3. Transmission performance comparison: (a) Q-factors vs. launch power per channel (dBm) at $3333 \mathrm{~km}$ and (b) Q-factors vs. transmission distance at optimum launch power for different schemes measured for the centre WDM channel $194 \mathrm{THz}(1545.32 \mathrm{~nm})$.

Fig. 3(a) and (b) shows the Q-factors versus signal launch power per channel and Q-factors versus transmission distance at optimum launch power respectively measured for the middle WDM signal at $194 \mathrm{THz}(1545.32 \mathrm{~nm})$ in three different dual-order counter-pumped schemes. The proposed scheme-2 shows the maximum Q-factor of $11.7 \mathrm{~dB}$ at optimum launch power per channel $(-2 \mathrm{dBm})$ with an improvement of $0.3 \mathrm{~dB}$ and $0.4 \mathrm{~dB}$ compared to conventional scheme-1 and 3 respectively. As all the schemes have similar signal power profiles as shown in Fig. 2(c), a similar noise performance is expected for all. However, a similar Q-factor enhancement in both linear and nonlinear regime proves the resulted improvement, due to the mitigation of signal RIN penalty in scheme-2. The RFL based scheme-1 shows the worst Q-factor of $11.3 \mathrm{~dB}$ at optimum launch power for the highest signal RIN penalty, whereas scheme-3 performs slightly better $(0.1 \mathrm{~dB})$ than scheme-1. In Fig. 3(b), the proposed scheme-2 provides maximum transmission distance up to $7915 \mathrm{~km}$ with $833 \mathrm{~km}$ reach extension compared to similarly performed $(\sim 7082 \mathrm{~km})$ scheme- 1 and 3 considering an HD-FEC threshold of $\mathrm{Q}=8.5 \mathrm{~dB}$. Q factors and received spectra at maximum transmission distances for all the Raman configurations are available at the conference.

\section{Conclusion}

We have demonstrated that, using only a $20 \mathrm{~mW}$ incoherently broadband seed in a dual-order counter-pumped DRA can significantly reduce the RIN transfer from higher order pumps to signal. This allows a minimum of $833 \mathrm{~km}$ transmission reach extension compared with conventional dual-order Raman counter-pumping schemes based on widely deployed low RIN semiconductor laser and cost-effective FBG assisted random fibre laser. Such broadband pump can provide flatter and wider gain spectrum which is useful and cost-effective for broadband transmission.

The work is funded by FP7 ITN programme ICONE (608099) and the EPSRC projects EP/J017582/1 and EP/L000091/1 (UNLOC and PEACE).

\section{References}

[1] S. Burtsev et al., Proc. OFC, p. 1-3, Los Angeles (2015).

[2] M. Tan et al., Opt. Express 23(17), 22181-22189 (2015).

[3] C. R. S. Fludger et al., J. Lightw. Technol. 19(8), 1140-1148 (2001).

[4] M. A. Iqbal et al., Proc. ECOC, P1.SC1.13, Gothenburg (2017).

[5] S. B. Papernyi et al., Proc. OFC, FB4, Anaheim (2002).

[6] D. V. Churkin et al., Phys. Rev. A, 82(3), pp. 033828, 2010. 